\title{
VIEWPOINT
}

\section{Point-of-Care Ultrasonography: Focused Versus Generalized Approaches to Scanning}

\author{
Michael Tanael, MD \\ Maxwell Air Force Base, Flight Medicine, United States Air Force, Montgomery, AL, USA.
}

$J$ Gen Intern Med 36(7):2115-6

DOI: $10.1007 / \mathrm{s} 11606-020-06290-3$

(c) Society of General Internal Medicine (This is a U.S. government work and not under copyright protection in the U.S.; foreign copyright protection may apply) 2020

$\mathrm{T}$ he use of diagnostic point-of-care ultrasonography (POCUS) by generalists has become increasingly common in recent years. Support for POCUS by influential internal medicine societies such as the American College of Physicians; Society of Hospital Medicine; and the Alliance for Academic Internal Medicine, coupled with the ubiquity of educational opportunities, has spurred its adoption by generalists and suggests it will become a requisite skill in the near future.

Traditionally, clinicians have conceived of diagnostic POCUS as a focused scan performed to answer a specific clinical question that arises from a patient's history or physical exam. ${ }^{1,2}$ Indeed, the majority of studies supporting the accuracy and usefulness of POCUS evaluate its ability to interrogate a single organ system or determine the presence/absence of a particular abnormality. ${ }^{3}$ However, as the enthusiasm for POCUS has grown, some experts have begun to advocate for expanding the use of POCUS, supporting its routine use for generalized assessments that interrogate several organ systems.

One protocol that has gained prominence has the clinician obtain parasternal, epigastric, apical/anterior, right upper quadrant, left upper quadrant, and suprapubic views (PEARLS) "whenever a thorough physical examination is indicated." Another protocol, the Ultrasound Screening Exam for Underlying Lesions (USEFUL), has the clinician scan the thyroid, heart, liver, gallbladder, aorta, kidneys, bladder, and prostate or uterus during routine patient encounters. ${ }^{5}$ While these protocols superficially resemble other well-validated systematic approaches to scanning such as the Focused Assessment with Sonography for Trauma (FAST) and Rapid Ultrasound for Shock and Hypotension (RUSH), they fundamentally differ from

Received July 17, 2020

Accepted October 5, 2020

Published online October 15, 2020 these assessments in that they divorce scanning from clinical history. Admittedly, POCUS does appear an effective screening tool in some well-defined asymptomatic patient groups such as in those deemed at risk for abdominal aortic aneurysm per the United States Preventive Services Task Force (USPSTF). ${ }^{3}$ However, regularly scanning several organ systems during routine patient encounters bears little resemblance to using POCUS to perform guideline-directed screenings.

These generalized protocols such as PEARLS and USEFUL may seem attractive as they provide a framework to consistently and efficiently perform a multitude of POCUS applications, but several studies show broad exams discover irregularities of varying clinical significance in a substantial minority of patients and have diminished accuracy compared with targeted exams. ${ }^{3}$ One representative study had family physicians evaluate asymptomatic patients with traditional physical exam at a periodic health evaluation and subsequently had another family physician perform an ultrasound-assisted physical exam on these patients. ${ }^{6}$ About $40 \%$ of the patients had abnormalities found on POCUS that traditional physical exam did not detect, but only $18 \%$ of these abnormalities required timely medical intervention.

Such results raise concerns that expanding the use of POCUS beyond targeted exams may lead to gratuitous downstream testing and medical care. Two decades ago, the authors of one of the studies mentioned above pointed out that POCUS can "[find] more abnormalities," but "whether it can improve outcomes...is yet to be determined." Not only does this scarcity of data showing improved outcomes persist, but in the intervening years since the authors made this observation, the results of several studies have cautioned against generalized ultrasound assessments - suggesting the support for broadening the scope of POCUS beyond its original conception stems from excitement about the technology, not a sober assessment of the medical literature; enthusiasm has outpaced the evidence. ${ }^{3}$

With its increased accuracy compared with the traditional physical exam, the concern that POCUS can lead to inferior care may reasonably meet some skepticism; however, an examination of data from South Korea gives credence to this concern. While ultrasonography has more accuracy than palpation in evaluation of the thyroid, its increased use in South Korea contributed to increased morbidity without improvements in mortality - a case study in the importance of careful consideration when 
integrating new technology into clinical practice to ensure medical advances improve patient care. ${ }^{7}$

In addition to concerns about accuracy and unnecessary medical care, a generalized POCUS exam requires more time to perform and document than a focused exam. The originators of PEARLS suggest it takes less than 3 minutes to perform, and the originators of USEFUL suggest it requires approximately 6 minutes to complete- but, other estimates for generalized POCUS exams suggest they entail larger investments of time. ${ }^{3-5}$ Regardless of the exact increase in the duration of a patient encounter, generalized exams have the potential to disrupt workflow. To increase efficiency and mitigate this disruption, some experts have proposed simultaneously performing a POCUS exam and taking a medical history. ${ }^{4}$ However, this approach may compromise the accuracy of scans as lack of attention to history serves as a major source of diagnostic error in ultrasonography. ${ }^{2}$

Diagnostic POCUS has the potential to improve quality of care by augmenting the traditional physical exam and will likely become a necessary skill for generalists to possess in the near future. However, as with any new technology, its implementation requires careful consideration to ensure maximization of its benefits and minimization of harms. The current body of evidence suggests expanding the use of POCUS to include routine generalized scans that assess several organ systems compromises accuracy, potentially leads to unnecessary medical care, and disrupts workflow. Consequently, clinicians ought to restrict their use of diagnostic POCUS to its traditional conceptualization as a focused exam that answers specific clinical questions arising from the history and physical.
Corresponding Author: Michael Tanael, MD; Maxwell Air Force Base, Flight Medicine, United States Air Force, Montgomery, AL, USA (e-mail: mtanael@gmail.com).

\section{Compliance with Ethical Standards:}

Conflict of Interest: The author declares that he does not have a conflict of interest.

Disclaimer: The views expressed are solely those of the author and do not reflect the official policy or position of the US Army, US Navy, US Air Force, the Department of Defense, or the US Government.

\section{REFERENCES}

1. Chawla TP, Cresswell M, Dhillon S, et al. Canadian Association of Radiologists Position Statement on Point-of-Care Ultrasound. Can Assoc Radiol J. 2019;70(3):219-225.

2. Pinto A, Pinto F, Faggian A, et al. Sources of error in emergency ultrasonography. Crit Ultrasound J. 2013;5(Suppl 1):S1.

3. Andersen CA, Holden S, Vela J, et al. Point-of-care ultrasound in general practice: a systematic review. Ann Fam Med. 2019;17:61-69.

4. Wagner M, Boughton J. PEARLS for an Ultrasound Physical and Its Routine Use as Part of the Clinical Examination. South Med J. 2018;111(7):389-394.

5. Steller J, Russell B, Lotfipour S, et al. USEFUL: Ultrasound Exam for Underlying Lesions incorporated into physical exam. West J Emerg Med. 2014;15(3):260-266.

6. Siepel T, Clifford DS, James PA, et al. The ultrasound-assisted physical examination in the periodic health evaluation of the elderly. J Fam Pract. 2000;49(7):628-632.

7. Vaccarella S, Franceschi S, Bray F, et al. Worldwide thyroid-cancer epidemic? The increasing impact of overdiagnosis. N Engl J Med. 2016;375(7):614-617.

Publisher's Note Springer Nature remains neutral with regard to jurisdictional claims in published maps and institutional affiliations. 